\title{
Modeling of W7-X superconducting coil cool-down using the 4C code
}

\author{
R. Bonifetto a, A. Kholiaa, B. Renardb, K. Rißec, L. Savoldi Richard ${ }^{a}$, R. Zanino ${ }^{a}$ \\ a Dipartimento di Energetica, Politecnico di Torino, I-10129 Torino, Italy \\ ${ }^{b}$ CEA, Irfu, SACM, Centre de Saclay, F-91191 Gif-sur-Yvette Cedex, France \\ ${ }^{c}$ Max-Planck Institut für Plasma Physik, EURATOM Association, D-17491 Greifswald, Germany
}

\begin{abstract}
The recently developed $4 \mathrm{C}$ thermal-hydraulic code is validated here against experimental data from the cooldown of a non-planar coil of the Wendelstein 7-X stellarator from room temperature to the superconducting transition temperature of $\sim 10 \mathrm{~K}$, performed during the cold test of the full set of coils at the cryomagnetic test facility of CEA Saclay. The computed results, i.e., temperature and mass flow rate evolutions in different coil components (helium, casing, etc.) are shown to be in good agreement with the experimental data. The simulations also show that during the transient the hot spot temperature inside the coil can be up to $\sim 3-4 \mathrm{~K}$ higher than what is seen on the casing surface.
\end{abstract}

Keywords: Fusion reactors, Superconducting coils, Simulation, Thermal-Hydraulics, Cool-down, W7-X.

\section{Introduction}

The Wendelstein 7-X (W7-X) stellarator is under construction at IPP Greifswald, Germany [1], [2]. The magnetic confinement of the plasma during the operation of W7-X will be based on 50 non-planar coils (NPCs, see figure 1); additionally, 20 planar coils surround the NPCs, allowing field adjustment and modification of the magnetic configuration. All W7-X coils are superconducting and based on a NbTi Cable in Conduit Conductor (CICC).

The cold test of all W7-X coils has been carried out in the CEA Saclay cryomagnetic test facility [3], requiring the cool-down from room temperature to the superconducting transition temperature $\mathrm{T}_{\mathrm{C}} \sim 10 \mathrm{~K}$ with supercritical helium. At any time during the cool-down transient, temperature differences between any two points of the coil are requested to stay below $40 \mathrm{~K}$, in order to limit thermo-mechanical stresses. In the cold test of a coil, the temperature difference between coolant inlet and outlet is monitored, together with the temperature of the coil casing at three different locations [3], but this measurement cannot obviously guarantee that any two

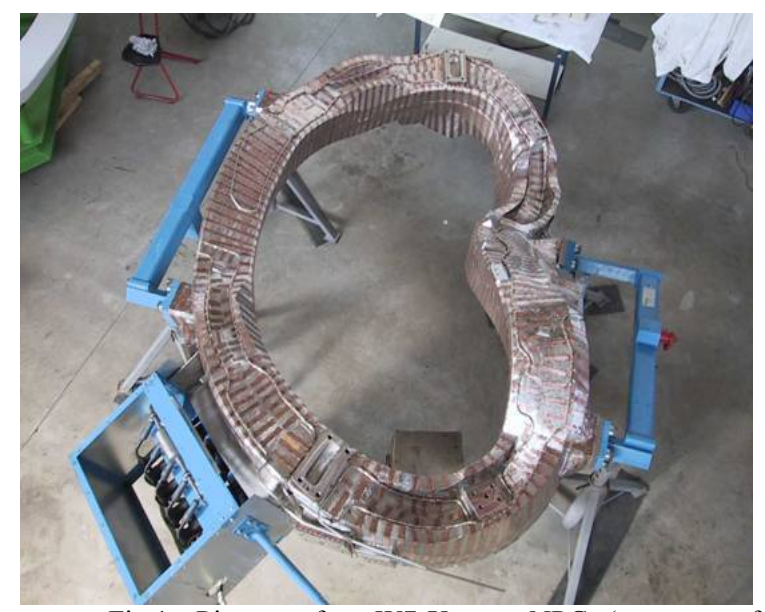

Fig.1. Picture of a W7-X non NPC (courtesy of Babcock Noell GmbH) points of the coil satisfy that constraint. In order to verify if this can be an issue, we apply the $4 \mathrm{C}$ thermal-hydraulic code [4], recently validated against ITER Toroidal Field Model Coil data for a safety discharge from $25 \mathrm{kA}$ [5], as well as applied to the quench analysis of an ITER TF coil [6] and to the temperature margin prediction in a JT60-SA TF coil [7], to the analysis of the cool-down of an NPC.

4C implements a model of compressible 1D supercritical helium flow in the entire winding and in the casing cooling channels (CCC), coupled with 3D heat conduction in the solid structures and fed, if needed, by an external cryogenic circuit which may include pumps, heat exchangers, cryolines, valves, etc. The simulation results are first compared to the experimental evolution of the measured variables, showing good capability to reproduce the experiment, depending mainly on the thermal resistance between casing and CCC. This represents the first validation of $4 \mathrm{C}$ against such (week) long transients, as well as for conditions ranging from room to cryogenic temperature. The code is then applied to the prediction of the maximum temperature differences between any two points of the coil.

\section{Problem definition}

The W7-X NPC is a very complex object, with a fully $3 \mathrm{D}$ structure, as can be seen from figure 1, where the path of the CCC (tortuous to avoid collisions with other components) is also clearly visible. The CCC are soldered on thin $(1 \mathrm{~mm})$ copper stripes realizing the heat transfer from the cooling pipes to the stainless steel (SS) casing through interrupted seam welding [8]. The picture also shows that the cross section of the NPC casing varies in the poloidal direction, having a series of connection plugs for the anchoring to the neighboring components in the machine. A certain degree of simplification of these features is however mandatory in order to make the model of the magnet cool down tractable and, at the same time, sufficiently flexible for the application to different coils, as explained in the following section.

Author's email: laura.savoldi@polito.it 


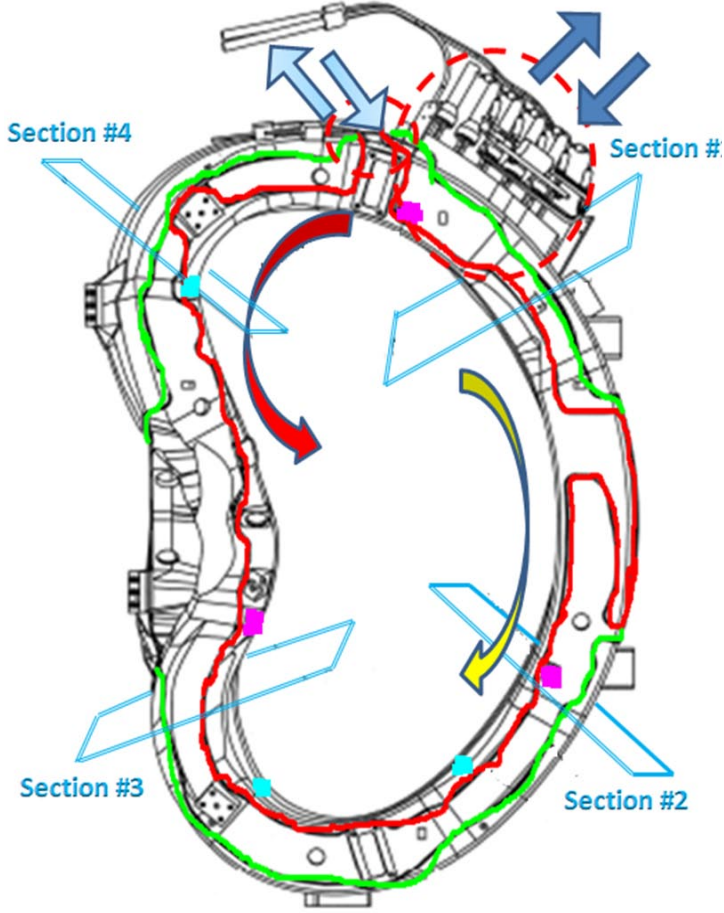

Fig.2. Sketch of the NPC cooling system highlighting 2D cut locations and numbering, as well as $\mathrm{He}$ inlet/outlet locations (dashed circles) in winding (blue arrows) and CCC (light blue arrows). The CCC are also shown (red line $=$ first turn of the first cooling channel, green line $=$ second turn of the second cooling channel), and the red arrow shows the flow direction in both. The He flow in the DLs is in the opposite direction (yellow arrow). The markers show the location of the thermometers on the casing surface (magenta $=$ Cernox, cyan $=$ Pt 100).

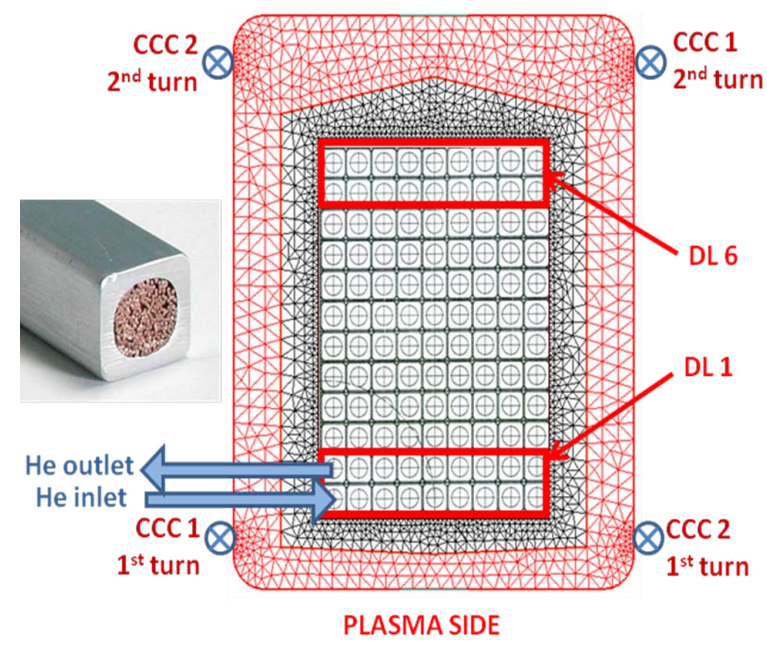

Fig.3. Geometry of a 2D cut and example of corresponding finite element discretization (casing $=$ red mesh, sand-epoxy embedding + ground insulation $=$ black mesh). The location used in the model for the two CCC (CCC1 and CCC2) is shown. The flow direction in these refers to, e.g., Section 4 seen from above. A cross section of the winding with the 6 DLs is also shown, together with $\mathrm{He}$ inlet and outlet locations for DL1. The W7-X CICC is shown in the inset.
Table 1: W7-X conductor geometrical parameters

\begin{tabular}{ll}
\hline Conductor area & $16 \times 16 \mathrm{~mm}^{2}$ \\
NPC DL hydraulic lengths & $152-170 \mathrm{~m}$ \\
Cable region diameter & $\sim 11.5 \mathrm{~mm}$ \\
Void fraction & $\sim 37 \%$ \\
Number of NbTi strands & 243 \\
Diameter of strands & $0.57 \mathrm{~mm}$ \\
$\mathrm{Cu} /$ nonCu & 2.6 \\
\hline
\end{tabular}

\subsection{Model}

The 3D structure of a W7-X NPC is discretized in the poloidal direction with a series of cuts as shown in figure 2. On each cut the transverse transient 2D heat conduction problem is solved by finite elements, coupled to transient 1D advection/diffusion in the third direction, along each double layer and along the CCC. For the sake of simplicity, all cuts have the same cross section shown in figure 3.

The W7-X NPC's are wound in double layers (DL's), using a rectangular NbTi CICC with Al jacket (6 DL's hydraulically in parallel, of $9 \times 2$ turns each) as shown in figure 3 . The main conductor data used for the present analysis are given in table conductor. The friction factor adopted for the conductors is given in [9], where it was deduced from the test of a large number of conductors and short samples using different fluids at both room and cryogenic temperature.

Two CCC, circular tubes with an inner diameter of 7 $\mathrm{mm}[10]$ and $\sim 19 \mathrm{~m}$ long, are wound in two turns each on the two lateral sides of the NPC, see figure 1 and figure 2 . The first turn of each (parallel) channel on the two different casing sides is located close to high magnetic field side of the coil ("plasma side" in figure 3), while the second turn moves on the opposite face of the casing and far away from the plasma side. These tortuous paths, see figure 1, are modeled in a simplified way, with the CCC at given (fixed) locations on the two lateral sides, see figure 3. The friction factor in the CCC is modeled as $f=M_{\mathrm{f}} \times$ $f_{\text {smooth }}$ where $f_{\text {smooth }}$ is the friction factor for a smooth circular tube and a multiplier $M_{\mathrm{f}}$ is applied in order to account for surface roughness and pressure losses due to sudden turns etc. $M_{\mathrm{f}}$ is then used as a parameter to fit the measured mass flow rate in the CCC.

Thermal coupling between the different coil components is either treated by finite element analysis of the heat conduction problem through a finite thickness, or lumped in the form of a thermal resistance. Both inter-turn and inter-layer thermal coupling have been modeled considering the thermal resistance (function of temperature) due to the glass-epoxy insulation $(0.6 \mathrm{~mm}$ turn insulation and $0.4 \mathrm{~mm}$ layer insulation, resulting into $1.2 \mathrm{~mm}$ inter-turn and $2.0 \mathrm{~mm}$ inter-layer, respectively) [11]. The parametric effect of the contact thermal resistance between adjacent layers of insulation [5] has been investigated. The thermal coupling between the casing and the CCC is modeled here without including the full geometrical detail of the copper stripes, but assuming instead, for the sake of simplicity, a continuous contact between the pipes and the casing. The heat transfer coefficient between the two is written 


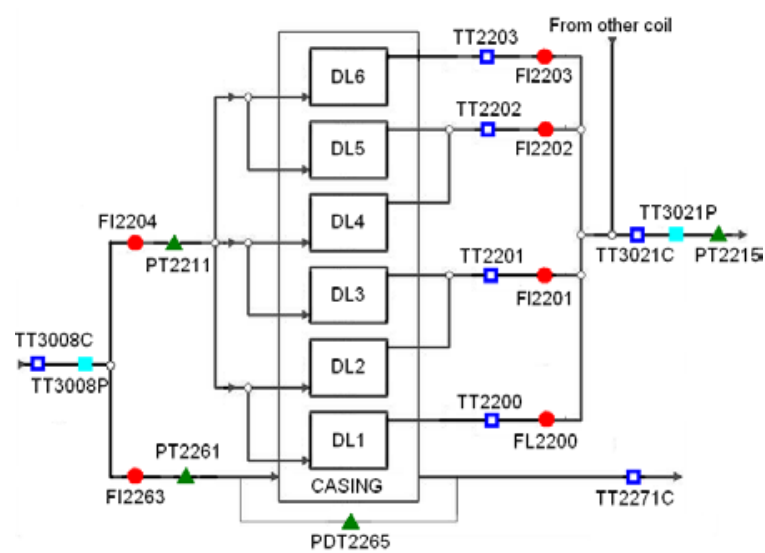

Fig.4. Cooling circuit of winding and casing with locations of selected sensors: Pt100 thermometers (solid cyan squares), Cernox thermometers (open blue squares), flow meters (solid red circles), pressure and pressure drop transducers (solid green triangles). Only one CCC is shown for simplicity.

as $\mathrm{h}_{\mathrm{Cc}}=\mathrm{M}_{\mathrm{h}} \times \mathrm{k}_{\mathrm{Cu}}(\mathrm{T}) / \delta_{\mathrm{Cu}}$ where $\mathrm{k}_{\mathrm{Cu}}(\mathrm{T})$ is the temperature-dependent heat conductivity of copper $\left(\mathrm{k}_{\mathrm{Cu}}\right.$ (a) $4 \mathrm{~K}$ ) was measured at $\sim 1500 \mathrm{~W} / \mathrm{m} \mathrm{K}$, which could correspond to RRR $>\sim 200), \delta_{\mathrm{Cu}}=1 \mathrm{~mm}$ is the thickness of the copper stripe and $\mathrm{M}_{\mathrm{h}}$ is a parameter needed because the real geometry of the contact between CCC and casing has not been modeled, for the sake of simplicity. The thermal coupling between case and winding occurs through a series of insulation layers, including the above-mentioned $1 \mathrm{~mm}$ inter-turn + interlayer, the $5 \mathrm{~mm}$ glass-epoxy (GE) ground insulation and the sand-epoxy winding embedding material (the latter is combined here with the ground insulation into a single material with GE properties, for the sake of simplicity).

As it can be seen in figure 3 , boundary conditions (BC) are needed at the outer surface of the casing. As a LN2 thermal shield surrounds the coil in the test, and is cooled down to $77 \mathrm{~K}$ with a time constant of $\sim 50 \mathrm{~h}$ together with the coil itself, radiation cooling in the initial part of the cool-down until the casing drops below $77 \mathrm{~K}$ is important; furthermore, conductive thermal bridges to the shield due to anchoring of the coil are also present. In order to include these contributions as a time dependent $\mathrm{BC}$ in our thermal model we follow the approach of [3], but evaluating these terms from the transient form of the first principle of thermodynamics $\mathrm{Q}_{\mathrm{rad}}+\mathrm{Q}_{\text {cond }}=\mathrm{dU} / \mathrm{dt}+\Sigma$ $(\mathrm{dm} / \mathrm{dt}) \Delta \mathrm{h}$, where the right hand side is estimated using measured temperatures and mass flow rates.

\subsection{Simulation setup}

We consider here the cool-down of the AAB10 coil performed in July 2007 [3]. We use the measured inlet pressure $(\sim 1 \mathrm{MPa})$ and temperature together with the outlet pressure as $\mathrm{BC}$ for our simulations, therefore without the need of modeling the external cryogenic circuit, while the remaining measurements (outlet temperatures, mass flow rates and casing surface temperatures) are used for the validation of the code, see figure 4 . It should be noted that at the coil outlet the global sensors (TT3021) come after the connection with the other coil circuit, so comparison with them can be only qualitative, while local Cernox thermometers (of which TT2201 and TT2202 are reliable) allow a quantitative comparison only at $\mathrm{T}<\sim 50 \mathrm{~K}$. The experimental $\mathrm{BC}$ have been smoothed (moving average on 100 points) and spikes have been removed in order to avoid abrupt changes in the computed quantities.

\section{Results}

Results are presented below for optimal (best-fitting) $\mathrm{M}_{\mathrm{f}}=2$ and two values $\mathrm{M}_{\mathrm{h}}{ }^{(1)}=0.00035$ and $\mathrm{M}_{\mathrm{h}}{ }^{(2)}=$ 0.0035 . Numerical convergence studies (not shown), prove the insensitivity of the computed solution to the main numerical parameters in the model. Reference values are: time step $\sim 10 \mathrm{~s}$, \# of nodes/DL $\sim 500$, \# of triangles/cut $\sim 3500$, \# of cuts $=4$.

The evolution of the cool-down is shown in figure 5 . The measured evolution of the $\mathrm{He}$ temperature at the outlet of the winding, see figure $5 \mathrm{a}$ is qualitatively well reproduced by the simulation, over the whole temperature range between $300 \mathrm{~K}$ and $\sim 10 \mathrm{~K}$. Quantitatively, discrepancies below $\sim 50 \mathrm{~K}$ with respect to TT2201, 2202
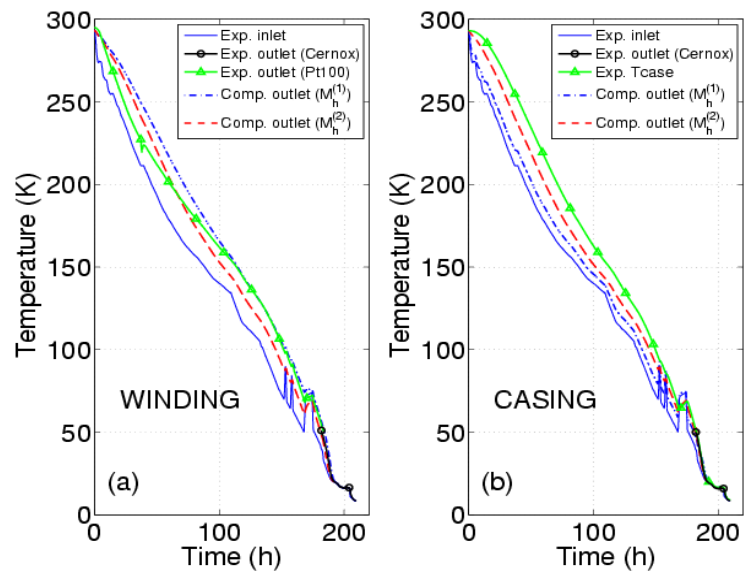

Fig.5. Evolution of common outlet He temperature: Experiment (symbols), computed with different $\mathrm{M}_{\mathrm{h}}$ (dashed, dash-dotted). The inlet He temperature is also shown (solid).
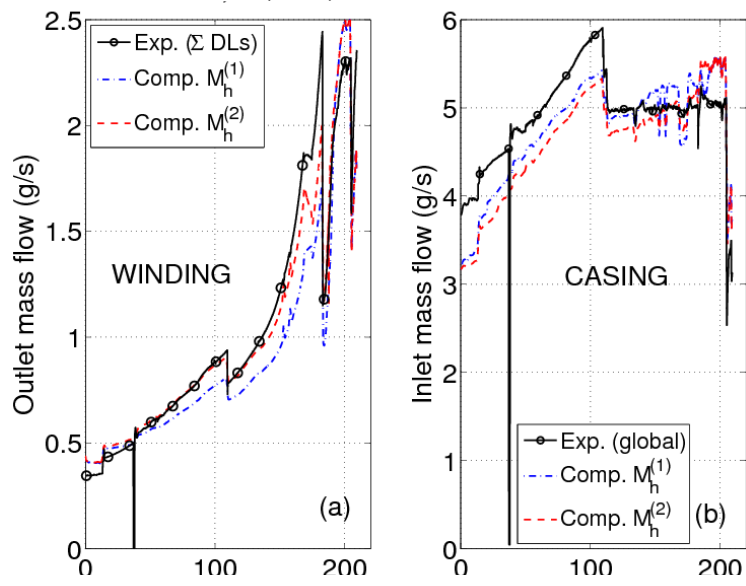

Fig.6. Global mass flow rate evolution: measured (circles), computed with different $\mathrm{M}_{\mathrm{h}}$ (red dashed, blue dash-dotted). 
are within $\pm 6 \mathrm{~K}$ (vs. $\pm 1 \mathrm{~K}$ measurement error bar), or \pm $15-20 \%$. As far as the outlet temperature of the CCC is concerned, see figure $5 \mathrm{~b}$, the simulation is nicely bracketed by the measured inlet temperature (obviously the lower bound) and by the measured casing surface temperature (which can be qualitatively used as upper bound) and it well reproduces the measured value (Cernox) in the cryogenic range (discrepancies below $\sim 50$ $\mathrm{K}$ are within $\pm 4 \mathrm{~K}$, or $\pm 10-15 \%$ ). Increasing $\mathrm{M}_{\mathrm{h}}$ leads to better coupling with the $\mathrm{CCC}$ and therefore higher $\mathrm{He}$ temperature at the casing outlet; this reduces the amount of heat to be extracted from the winding and therefore leads to a lower outlet temperature from the winding.

Figure 6 shows a good agreement (within $\sim 10 \%$ ) between measured and computed mass flow rates in the casing (consequence of the best fitting choice of $\mathrm{M}_{\mathrm{f}}$ ), whereas the agreement in the winding is still acceptable with discrepancies of the order of $\sim 10-30 \%$ (vs. $5 \%$ measurement error bar), which is the claimed accuracy of the correlation [9] we are using here. The effect of $\mathrm{M}_{\mathrm{h}}$ in both winding and $\mathrm{CCC}$ directly reflects the effect on the temperature (see figure 5), combined with the temperature dependence of density and viscosity.

The maximum temperature difference measured during the cool-down (defined in the experiment as the difference between the hottest temperature sensor on the casing and the helium inlet temperature) is shown in figure 7: the measured evolution is bracketed by the simulation results with different $\mathrm{M}_{\mathrm{h}}$, confirming the consistency of the analysis. Independently of the value of $\mathrm{M}_{\mathrm{h}}$, the simulations capture the qualitative features of the experimental trace.

The computed results for the maximum (i.e., over the whole coil) temperature difference during the cool-down are also reported in figure 7. One sees that, independently of the value of $M_{h}$, the computed hotspot can be up to $\sim 3-4 \mathrm{~K}$ above the computed casing surface temperature; indeed, according to the simulation the hot spot location moves during the cool-down and is located on the casing surface only for a limited portion of the transient, see figure 8 left. In figure 8 right the main

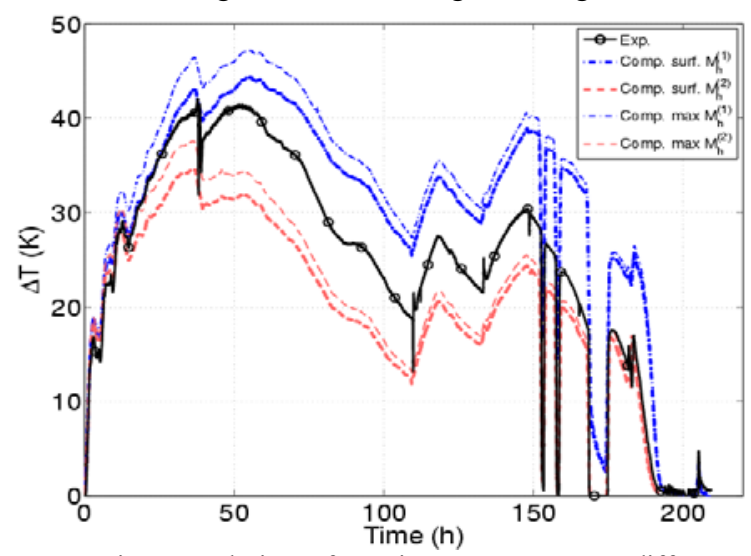

Fig.7. Evolution of maximum temperature difference between casing surface (at sensor locations only) and He inlet: measured (circles), computed with different $\mathrm{M}_{\mathrm{h}}$ (thick red dashed, thick blue dash-dotted). The computed absolute maximum temperature difference is also shown (thin lines).
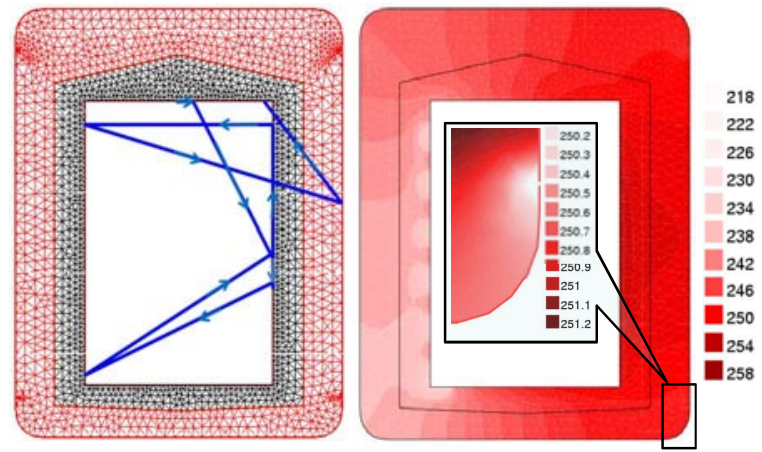

Fig.8. Left: Computed trajectory on the coil cross section of the maximum temperature location. Right: Computed temperature distribution $(\mathrm{K})$ on Section \# 1 at $\mathrm{t} \sim 35$ h. $\mathrm{M}_{\mathrm{h}}=\mathrm{M}_{\mathrm{h}}^{(2)}$.

cooling mechanisms active during this transient (DL and CCC He flow) are clearly highlighted by the computed 2D temperature map.

\section{Conclusions and perspective}

The validation of the $4 \mathrm{C}$ code against experimental data from the cool-down of a non-planar coil of the W7$\mathrm{X}$ stellarator during its cold test has been presented. It was shown that the $4 \mathrm{C}$ code is able to reproduce the evolution of the main measured quantities ( $\mathrm{He}$ and structure temperatures, He mass flow rates) with a good level of accuracy, depending mainly on the thermal coupling between casing and CCC.

The code shows that during the cooldown the maximum temperature difference between any two points inside the coil might have exceeded, by up to $\sim 3$ $4 \mathrm{~K}$, the value measured by the available diagnostics on the coil surface.

The 4C code is now validated both against fast (safety discharge) and slow (cool-down) transients. In perspective, the code will be applied to the analysis of thermal-hydraulic transients in the ITER coils.

\section{References}

[1] K. Riße, Fus. Eng. Des. 84, 1619 (2009).

[2] L. Wegener, Fus. Eng. Des. 84, 106 (2009).

[3] B. Renard et al., Fus. Eng. Des. 84, 1588 (2009).

[4] L. Savoldi Richard et al., Cryogenics 50, 167 (2010).

[5] R. Zanino et al., "Validation of the 4C Thermal-Hydraulic Code against $25 \mathrm{kA}$ Safety Discharge in the ITER Toroidal Field Model Coil (TFMC)", presented as paper 2-LZ-06 at ASC, Washington DC, 2010, to appear in IEEE Trans. Appl. Supercond. (2011).

[6] R. Zanino et al., Fus. Eng. Des. 85, 752 (2010).

[7] R. Bonifetto et al., "Computation of JT-60SA TF coil temperature margin using the $4 \mathrm{C}$ code", presented at SOFT, Porto, 2010, submitted to Fus. Eng. Des..

[8] M. Nagel and F. Schauer, "Cooling of the W7-X Superconducting Coils", Proceedings ICEC 19, pp. 677-680 (2002).

[9] M. Wanner, "Pressure Drop of the W7-X Cable-in-Conduit Conductor ", AST_D_2234VD1.1 Internal report, Fusion For Energy, Broader Fusion Development Department, Garching (Germany) (2009).

[10] F. Schauer, Fus. Eng. Des. 66-68 1045 (2003).

[11] L. Savoldi et al, Cryogenics 40, 179 (2000). 